Open Access

\title{
Uridine supplementation exerts anti- inflammatory and anti-fibrotic effects in an animal model of pulmonary fibrosis
}

Sanja Cicko ${ }^{1}$, Melanie Grimm ${ }^{1}$, Korcan Ayata ${ }^{1}$, Jessica Beckert ${ }^{1}$, Anja Meyer ${ }^{1}$, Madelon Hossfeld ${ }^{1}$, Gernot Zissel ${ }^{1}$, Marco Idzko ${ }^{1 *}$ and Tobias Müller ${ }^{1,2^{*}}$

\begin{abstract}
Rationale: Pulmonary fibrosis is a progressive disease with only few treatment options available at the moment. Recently, the nucleoside uridine has been shown to exert anti-inflammatory effects in different animal models, e.g. in acute lung injury or bronchial asthma.

Method: Therefore, we investigated the influence of uridine supplementation on inflammation and fibrosis in the classical bleomycin model. Male C57BL/6 mice received an intratracheal injection of bleomycin on day 0 and were treated intraperitoneally with uridine or vehicle. The degree of inflammation and fibrosis was assessed at different time points.

Results: Uridine administration resulted in attenuated inflammation, as demonstrated by reduced leukocytes and pro-inflammatory cytokines in the broncho-alveolar lavage (BAL) fluid. Furthermore, collagen deposition in the lung interstitium was also reduced by uridine supplementation. Similar results were obtained in a model in which animals received repeated intraperitoneal bleomycin injections. In addition uridine inhibited collagen and TGF- $\beta$ synthesis by primary lung fibroblasts, the release of pro-inflammatory cytokines by human lung epithelial cells, as well as the production of reactive oxygen species by human neutrophils.

Conclusion: In summary, we were able to show that uridine has potent anti-inflammatory and anti-fibrotic properties. As uridine supplementation has been shown to be well tolerated and safe in humans, this might be a new therapeutic approach for the treatment of fibrotic lung diseases.
\end{abstract}

\section{Background}

Idiopathic pulmonary fibrosis (IPF) is a disease of unknown origin characterized by progressive loss in lung function leading to respiratory failure with currently no effective treatment options available and therefore a poor prognosis. The pathophysiology of IPF is not fully understood yet. However, injury of alveolar epithelial cells (AECs) paralleled by oxidative stress leading to abnormal activation of fibroblasts is considered to be crucial in this context. The formation of fibroblast and myofibroblast foci via the proliferation of lung fibroblasts, the recruitment of circulating fibrocytes, and epithelial to mesenchymal

\footnotetext{
* Correspondence: marco.idzko@uniklinik-freiburg.de; tobmueller@ukaachen.de 'Department of Pneumology, University Hospital Freiburg, Killianstrasse 6, 79106 Freiburg, Germany

Full list of author information is available at the end of the article
}

transition hereby depends on mediators secreted by activated AECs. In summary, these processes result in excessive deposition of collagen, fibronectin and other components of the extracellular matrix in the lung parenchyma [1-3].

Besides their well-characterized role in cell metabolism, the involvement of different nucleosides in particular adenosine in different inflammatory disorders has been demonstrated extensively. Adenosine-induced overproduction of IL-13 results in pulmonary fibrosis, respiratory failure and death in a transgenic mouse model [4]. Inosine in contrast has been found to be protective in animal models of acute lung injury or bronchial asthma in an adenosine receptor-dependent manner [5, 6]. In contrast no receptor has been identified for the nucleoside uridin so far which has also been demonstrated to have anti-inflammatory properties [7]. Recently we were able to show that the exogenous application of uridine results 
in decreased allergic airway inflammation in both OVA- and house dust mite induced asthma. Though the mechanisms behind these findings could not be fully resolved an effect on lung epithelial cells could be shown [8]. Additionally, uridine is a potent inhibitor of leucocyte adhesion [9]. Interestingly, oral uridine supplementation is a safe, well tolerated and efficacious treatment to reduce mitochondrial toxicity caused by highly active antiretroviral therapy (HAART) in humans $[10,11]$.

In this study we investigated therapeutic properties of uridine in the classical animal model of bleomycininduced lung injury and fibrosis.

\section{Material and methods}

Animals

C57Bl/6 mice, were bred at the animal facility at the University of Freiburg. All experiments were performed according to institutional guidelines of the local animal ethics committee (Regierungspräsidium Freiburg).

Bleomycin model of pulmonary fibrosis (intratracheal model) Male C57BL/6 animals were anaesthetized by i.p. ketamine/xylazine administration and received an intratracheal (i.t.) injection of bleomycin $(80 \mu \mathrm{l}, 1 \mathrm{mg} / \mathrm{ml})$ or vehicle (saline) as a negative control. Animals were treated with uridine ( $80 \mu \mathrm{l}, 24 \mu \mathrm{g} / \mathrm{ml}$; Sigma Aldrich, Germany) or vehicle at the indicated time points. Mice were sacrificed at different time points (see results section) via i. p. injection of thiopental. BAL was performed with $3 \times 1 \mathrm{ml}$ of $\mathrm{Ca}^{2+}$ and $\mathrm{Mg}^{2+}$ free PBS (Gibco, Paisley, UK) supplemented with 0.1 mM sodium EDTA (Sigma Aldrich, Germany), followed by lung resection and storage in OCT freezing medium. BAL cells were counted, differential cell counts were done by FACS analysis, as described previously [12]. Frozen lung sections were stained with hematoxylin and eosin for histological analysis.

\section{Bleomycin model of pulmonary fibrosis (intraperitoneal model)}

Male C57BL/6 animals received i.p. injections of bleomycin $(140 \mu \mathrm{l}, 6 \mathrm{mg} / \mathrm{ml})$ or vehicle (saline) as a negative control twice a week over 4 weeks. Starting from day 14 on animals were treated intraperitoneally with uridine (200 $\mu \mathrm{l}, 2,4 \mathrm{mg} / \mathrm{ml}$ ) or vehicle 3 times a week. On day 30 animals were killed and BAL was performed and analysed as described above. Frozen lung sections were stained with hematoxylin and eosin for histological analysis.

\section{Mediator measurements in BALF}

BALF cytokine contents were determined by ELISA (R\&D Systems, Minneapolis, USA), as described by the manufacturer. BALF collagen content was measured by Sircol assay (Biocolor, Carrickfergus, UK).

\section{Collagen quantification in histological lung slides}

Frozen lung sections were incubated in picrosirius red solution (0,2 gr of Picosirius Red diluted in $100 \mathrm{ml}$ of $1,2 \%$ picric acid, both Sigma-Aldrich), for one hour. After washing with water, tissue sections were stained with hematoxylin for 5-10 s. Slides were washed with running tap water and dehydrated in $70 \%, 90 \%$ and absolute ethanol, followed by xylene. Entellan (Merck) was used to mount the coverslip. Images were obtained using Axio Lab.A1 microscope (Zeiss) with 200× magnification and AxioCam ICc1 (Zeiss). Collagen quantification was made with ImageJ.

\section{Cell culture}

A549 cells were grown in RPMI1640-Medium (Gibco, Paisley, UK) containing $10 \%$ fetal calf serum (FCS; Biochrom, Berlin, Germany) and $1 \%$ penicillin/streptomycin (P/S; Gibco, Paisley, UK) in cell culture flasks (BD Falcon, Bedford, MA) at $37{ }^{\circ} \mathrm{C}, 5 \% \mathrm{CO} 2$, and $100 \%$ humidity. For subculture cells were trypsinized and seeded into 24-well tissue culture plates at a density of $2 \times 10^{5}$ cells/ well. After $2 \mathrm{~h}$, medium was changed and cells were stimulated as indicated. After additional $8 \mathrm{~h}$, cell supernatants were collected and analyzed by ELISA.

For the isolation of primary lung fibroblasts lungs of $\mathrm{C} 57 \mathrm{Bl} / 6$ mice were excised and cut in small pieces, followed by digestion with collagenase. Single cell suspensions were obtained by passing through a cell strainer. Cells were grown in RPMI1640-Medium (Gibco, Paisley, UK) (supplemented $10 \% \mathrm{FCS}$ and $1 \% \mathrm{P} / \mathrm{S}$ ) in cell culture flasks (BD Falcon, Bedford, $\mathrm{MA}$ ) at $37{ }^{\circ} \mathrm{C}, 5 \% \mathrm{CO}_{2}$ in a humidified atmosphere. Medium was changed every 2 or 3 days. Cells were trypsinized after 14 days and used for experiments. Purify of isolated cells was assessed by microscopy. The cells were seeded into 6-well tissue culture plates at a density of $0,5 \times 10^{6}$ cells/well. After $24 \mathrm{~h}$, medium was changed and cells were stimulated with Bleomycin $(1 \mu \mathrm{g} / \mathrm{ml}) \pm$ uridine. After $24 \mathrm{~h}$, total RNA of these cells was isolated with Qiazol (QIAGEN GmbH, Hilden, Germany) according to the manufacturer's protocol.

\section{Isolation of human neutrophils and measurement of reactive oxygen species (ROS)}

Neutrophils from healthy volunteers were isolated from venous blood using a Pancoll gradient (PAN-Biotech $\mathrm{GmbH}$, Aidenbach, Germany) as described previously [13]. Resulting neutrophils were resuspended in PBS. Neutrophils were seeded in 96-well plates $\left(1 \times 10^{5} /\right.$ well $)$, in RPMI1640 medium supplemented with $10 \%$ FCS and penicillin/ streptomycin, and incubated at $37{ }^{\circ} \mathrm{C}, 5 \% \mathrm{CO} 2$ in a humidified atmosphere. Neutrophils were preincubated with uridine $\left(10^{-5} \mathrm{~mol} / \mathrm{l}\right)$ or vehicle for $15 \mathrm{~min}$, then $50 \mu \mathrm{l}$ of lucigenin $(2 \mathrm{mg} / \mathrm{ml})$ was added. Finally, $10 \mu \mathrm{l}$ of Bleomycin $(1 \mu \mathrm{g} / \mathrm{ml})$ were added to the cells and the stimulated generation of ROS was measured by a computerized 
chemoluminometer at $37{ }^{\circ} \mathrm{C}$ (Berthold Technologies LP96V, Germany).

\section{Quantitative PCR}

Quantitative PCR was performed on LightCyler 480 (Roche) using the fast-blue + UNG kit (Eurogentec). Collagen, TGF- $\beta$ and $ß 2$-microglobulin primers/probes were designed using Beacon Designer v 7.50 (Premier Biosoft). Percent reference gene (RG) values for the gene of interest (GOI) were calculated using the formula: $\% R G=100 \times 2(-\Delta C t)$. Cumulative standard deviations were calculated using the formula: $\mathrm{SD}=100 \times 2(-\Delta \mathrm{Ct}) \times$ $((\ln 2 \times \mathrm{SDRG}) 2+(\ln 2 \times \mathrm{SDGOI}) 2) 1 / 2[12]$.

\section{Statistical analysis}

If not stated otherwise, groups were compared using anova, followed by Bonferroni comparison test. Probability values of $p<0.05$ were regarded as significant.

\section{Results}

Uridine supplementation decreases inflammation in the early phase after intratracheal bleomycin administration To investigate the influence of uridine supplementation on the course of fibrotic lung disease we used the well-established animal model of bleomycin induced lung injury/fibrosis. In the prophylactic protocol mice received an intratracheal injection of bleomycin or vehicle on day 0 . Animals were treated intraperitoneally with uridine or vehicle after $6 \mathrm{~h}$, on day 5 and on day 10 following bleomycin administration. Animals were sacrificed on day 7 and day 14 and assessed for inflammation and fibrosis. Treatment with uridine resulted in decreased inflammation, as shown by reduced inflammatory cells in the broncho alveolar lavage (BAL) fluid (Fig. 1a). This was paralleled by decreased BAL fluid cytokine levels and by attenuated inflammation on histological lung sections (Fig. 1b, c). In addition, the collagen content in the BAL fluid was lower in the animals treated with uridine (Fig. 1d).

\section{Uridine supplementation decreases inflammation and fibrosis in the late phase after intratracheal bleomycin administration}

As uridine was able to decrease inflammation and fibrosis when administered early after bleomycin we next questioned whether uridine administration is also protective during the fibrotic phase. Therefore, bleomycin or vehicle was injected intratracheally on day 0 . Starting from day 14 mice were treated 3 times a week with either uridine or vehicle. The degree of inflammation and fibrosis was determined on day 30. As shown in Fig. 2a, uridine treatment significantly reduced total BAL cell count and the number of macrophages and lymphocytes. Additionally, the concentration of pro-inflammatory and pro-fibrotic cytokines was lower in the BAL fluid derived from uridine- treated animals (Fig. 2b). Furthermore, we observed reduced collagen deposition in the airspaces and decreased collagen levels in the BAL fluid (Fig. 2c, d).

\section{Uridine supplementation inhibits inflammation and fibrosis following chronic bleomycin administration}

In contrast to IPF in humans, pulmonary fibrosis after a single intratracheal bleomycin administration is known to be self-limiting. Hence, another model in which mice were repeatedly exposed to intraperitoneal bleomycin was used. This model is characterized by long lasting fibrosis and subpleural scaring, a pattern similar to usual interstitial pneumonia (UIP) in humans [14]. Therefore, animals received intraperitoneal bleomycin injections twice a week over 4 weeks. Treatment with uridine which was started at day 15 was associated with a strong reduction in inflammation as demonstrated by reduced inflammatory cells and pro-inflammatory cytokines in the BAL fluid (Fig. 3a, b). Moreover, histological analysis showed decreased tissue fibrosis paralleled by lower collagen contents in the BAL fluid of uridine treated animals (Fig. 3c, d).

\section{Uridine inhibits the release of pro-inflammatory mediators by human lung epithelial cells}

Alveolar epithelial cells are known to play a pivotal role in the pathogenesis of fibrotic lung diseases [1]. In pursuit of the mechanisms behind our findings we investigated the effects of uridine on the production of the pro-inflammatory cytokines IL- 6 and IL- 8 by A549 cells, a cell line resembling human alveolar epithelial cells type 2. As shown in Fig. 4, uridine was able to suppress the bleomycin induced release of both IL- 6 and IL-8 in a dose dependent manner.

\section{Uridine inhibits collagen and TGF- $\beta$ production by primary lung fibroblasts}

Excessive production and deposition of extracellular matrix components by lung fibroblasts is crucial for the development of pulmonary fibrosis. In addition fibroblasts interact with inflammatory and lung structural cells and are capable of releasing different cytokines and growth factors [2]. As demonstrated in Fig. 5, uridine decreased collagen and TGF- 3 mRNA synthesis by primary lung fibroblasts.

\section{Uridine inhibits production of reactive oxygen species by human neutrophils}

The importance of oxidative stress in the pathophysiology of pulmonary fibrosis has been demonstrated extensively [2]. Thus we sought to determine whether uridine can influence the production of reactive oxygen species by human neutrophils. As shown in Fig. 6, exposure to bleomycin resulted in transient ROS production. However, pre-treatment with uridine significantly inhibited ROS production by neutrophils. 


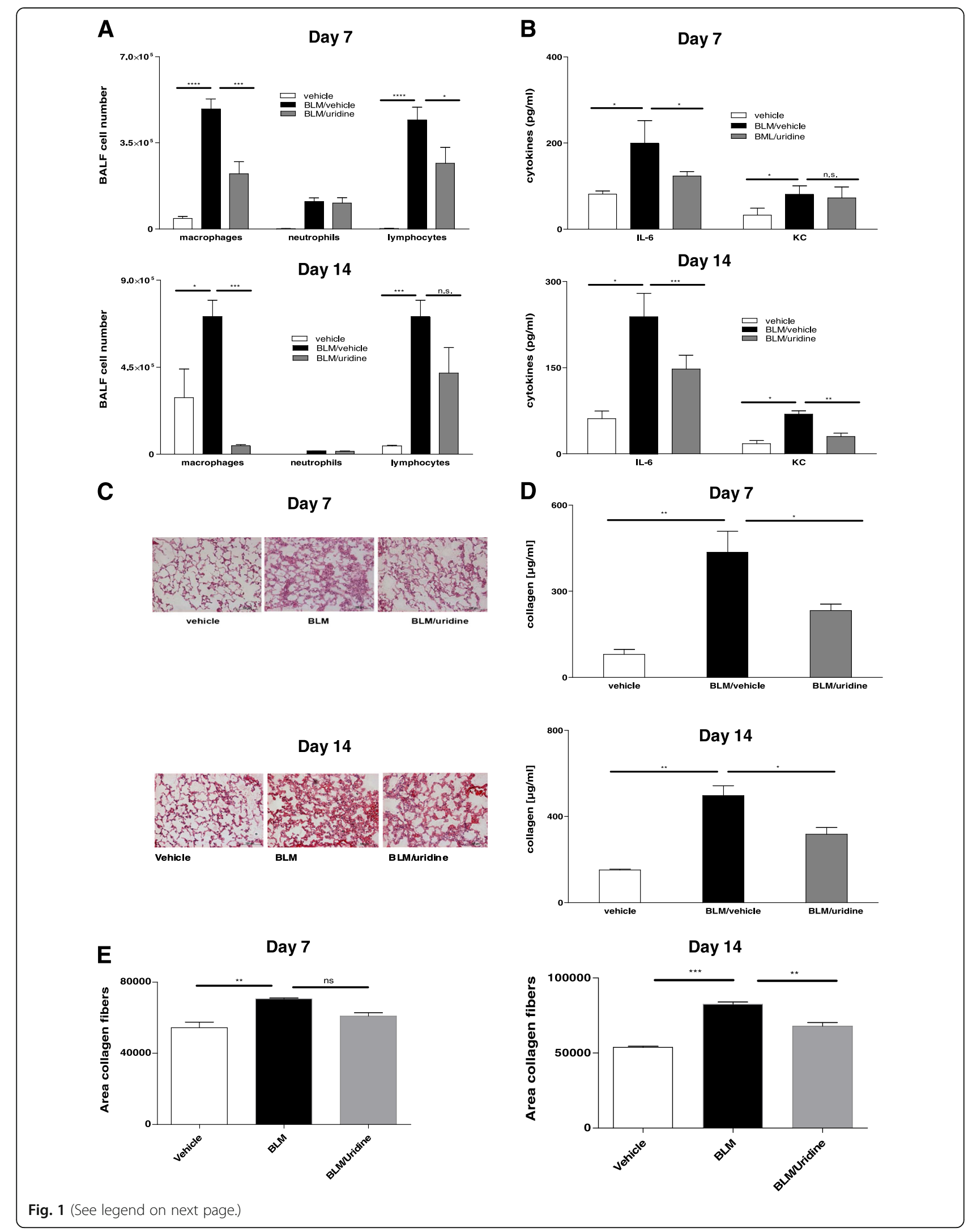


(See figure on previous page.)

Fig. 1 Uridine supplementation decreases inflammation in the early phase after intratracheal bleomycin administration. Animals were treated with i. t. bleomycin on day 0 . Uridine or vehicle was administered after 6 h, at day 5 and at day 10 . Animals were assessed for inflammation and fibrosis at day 7 and day 14. a BALF differential cell counts. b BALF cytokine levels. $\mathbf{c}$ HE stainings of lung sections. d BALF collagen content. e Collagen content on histological lung slides. Data are means \pm SEM, $n=4-6$ mice per group. ${ }^{*} p<0.05 ;{ }^{* *} p<0.01 ;{ }^{* * *} p<0.001 ;{ }^{* * *} p<0,0001$. The experiment was repeated twice with similar results

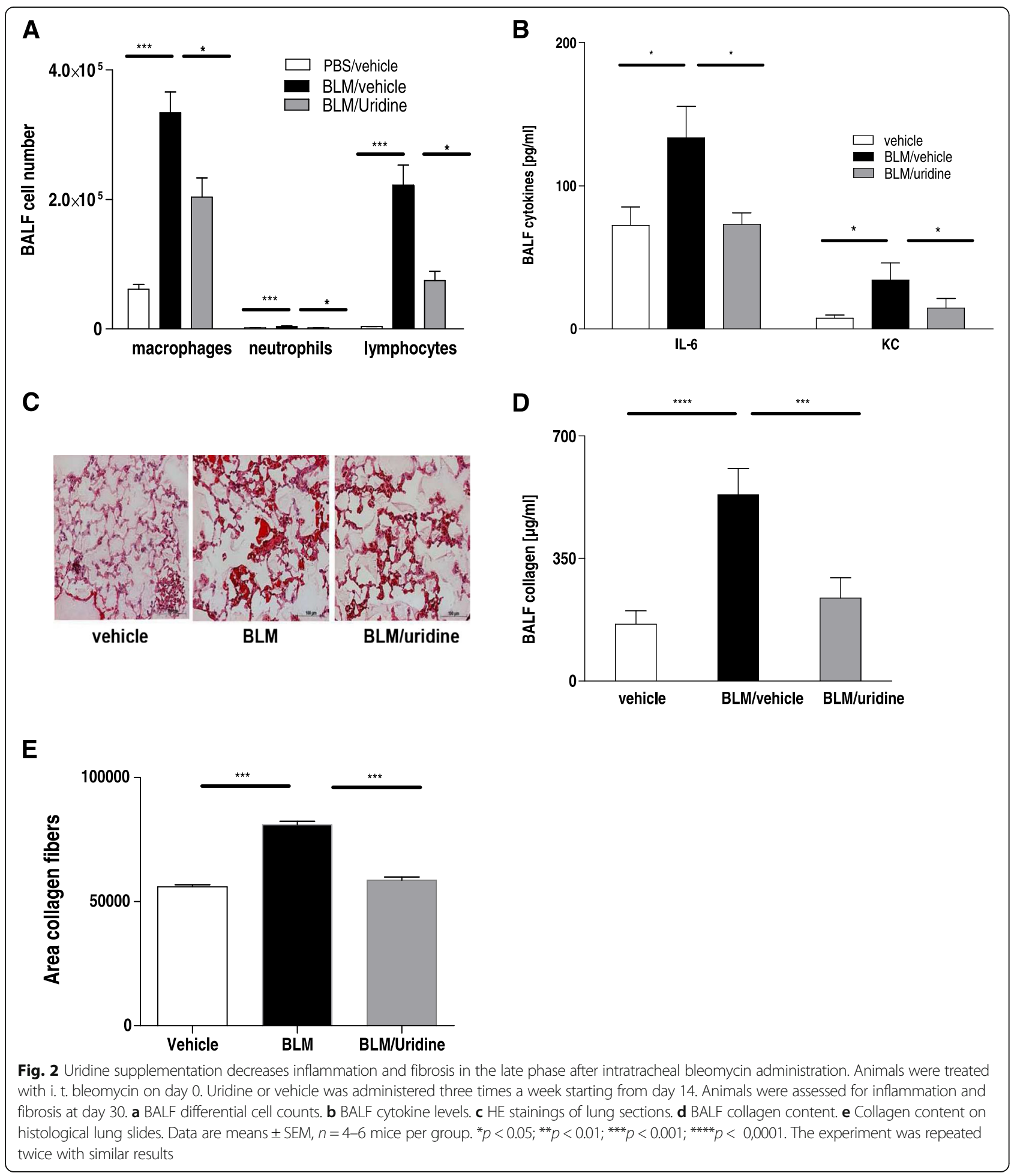




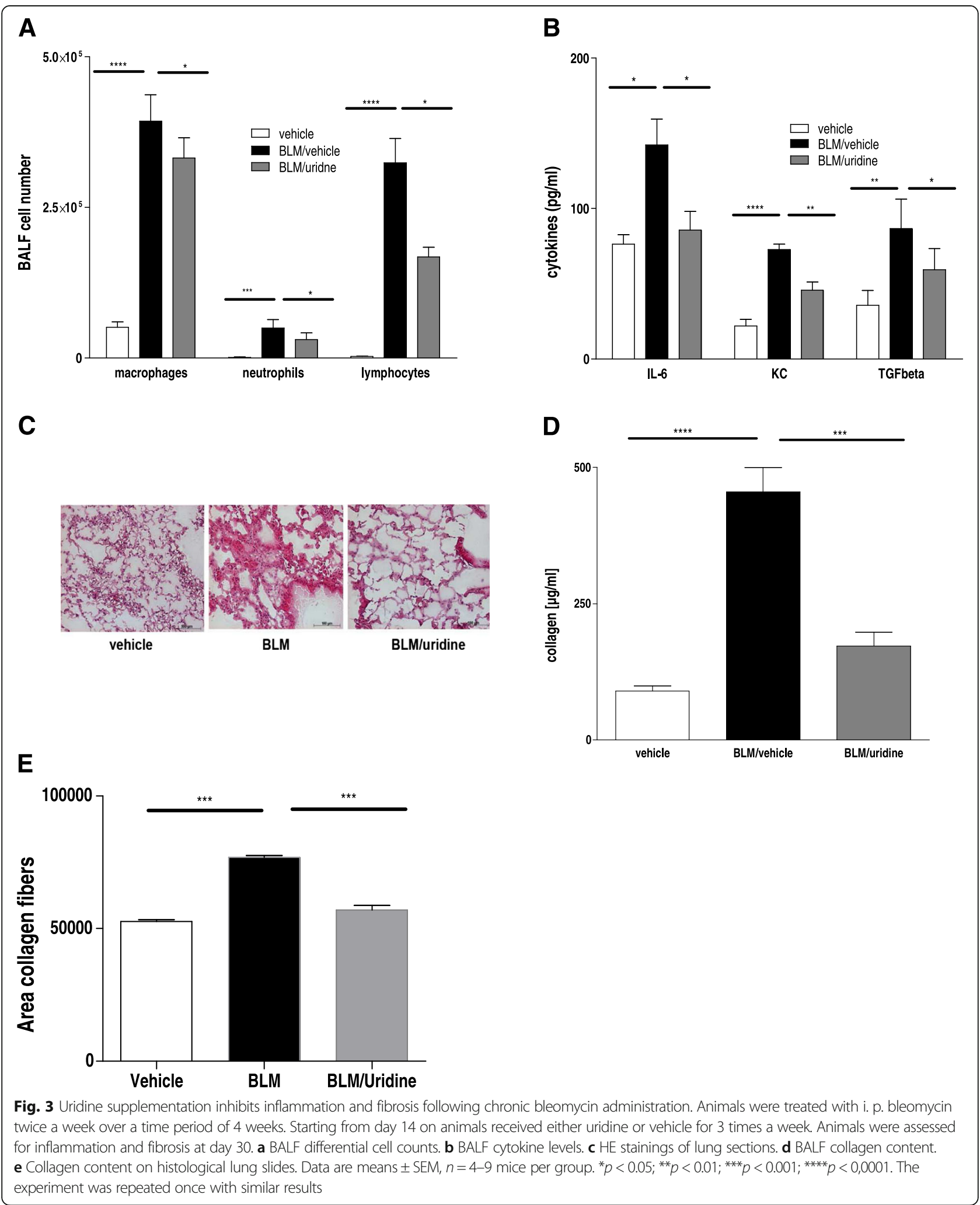

\section{Discussion}

The involvement of the nucleoside adenosine in the pathogenesis of inflammatory lung disorders, including pulmonary fibrosis has been studied extensively $[4,15,16]$. Recently, we were able to demonstrate that local administration of uridine inhibited the cardinal features of 

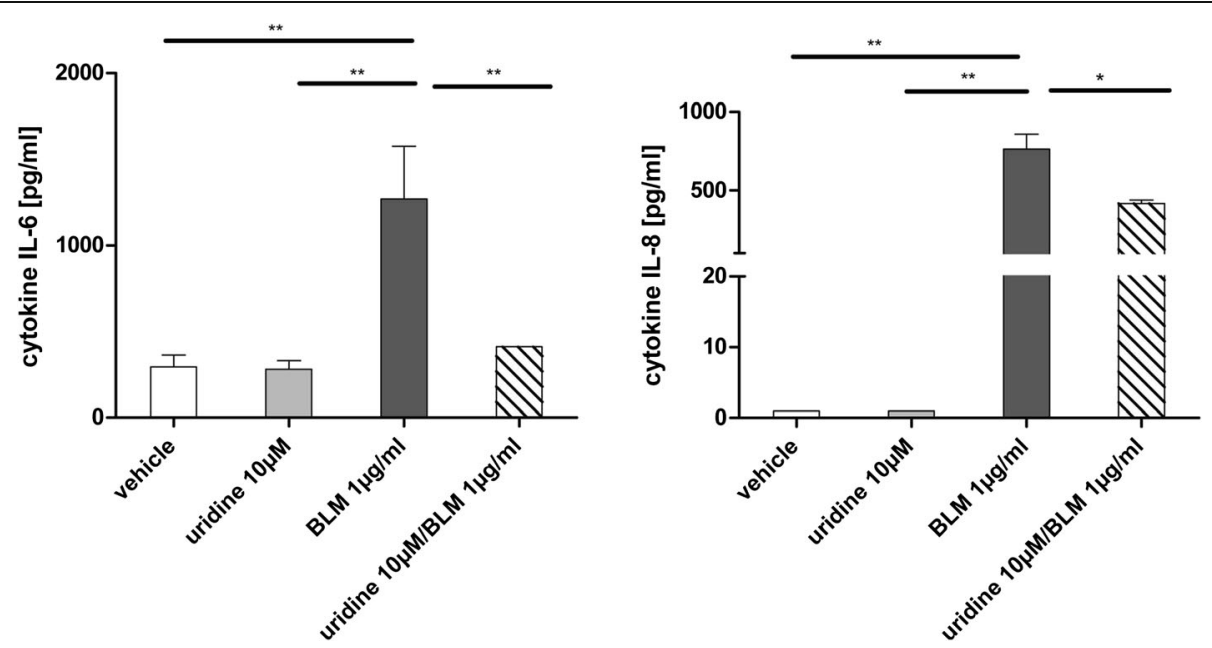

Fig. 4 Uridine inhibits the release of pro-inflammatory mediators by human lung epithelial cells. A549 cells were stimulated with bleomycin (1 $\mu \mathrm{g} / \mathrm{ml})$ \pm uridine. After an incubation period of $24 \mathrm{~h}$ levels of IL- 6 and IL-8 in cell culture supernatants were determined by ELISA. Data are means \pm SEM, $n=3$. ${ }^{*} p<0.05 ;{ }^{* *} p<0.01$

bronchial asthma [8]. In this study we investigated whether uridine supplementation can also influence the course of fibrotic lung disease.

In an acute model of bleomycin induced lung injury intraperitoneal treatment with uridine resulted in decreased inflammation and collagen deposition. These results are in line with previous studies showing that uridine administration is protective in Sephadex- or allergen-induced lung inflammation $[7,8,17]$. However, beyond dampening inflammation following acute bleomycin administration uridine was also able to suppress tissue fibrosis at later time points. Furthermore, fibrotic changes were also reduced by uridine when bleomycin was administered repeatedly. This animal model is characterized by longer lasting fibrosis with subpleural predominance. Hence it is supposed to be closer to idiopathic pulmonary fibrosis in humans than the acute model [14]. To our best knowledge this is the first study showing that uridine has both anti-inflammatory and anti-fibrotic properties.

A receptor specific for uridine has not been identified, yet. Consequently, it has been hypothesized that uridine might exert its effects indirectly via the generation of UDP and UTP being able to bind to the purinergic receptors $\mathrm{P}_{2} \mathrm{Y}_{2}, \mathrm{P}_{2} \mathrm{Y}_{4}$, and $\mathrm{P} 2 \mathrm{Y}_{6}$. However, both $\mathrm{P}_{2} \mathrm{Y}_{2}$ and $\mathrm{P} \mathrm{Y}_{6}$ receptor subtypes have actually been demonstrated to mediate pro-inflammatory effects in bronchial asthma or chronic obstructive pulmonary disease $[12,18,19]$. Another possibility might be the interaction of uridine with adenosine receptors. In accordance with this a previous study demonstrated that uridine can actually activate $\mathrm{A}_{1}$ receptors [20]. Though there is evidence that $A_{1}$ receptors exhibit both pro- and anti-inflammatory activities, most studies suggest that the activation of $A_{1}$ receptors is in summary pro-inflammatory [21, 22]. Moreover, deficiency

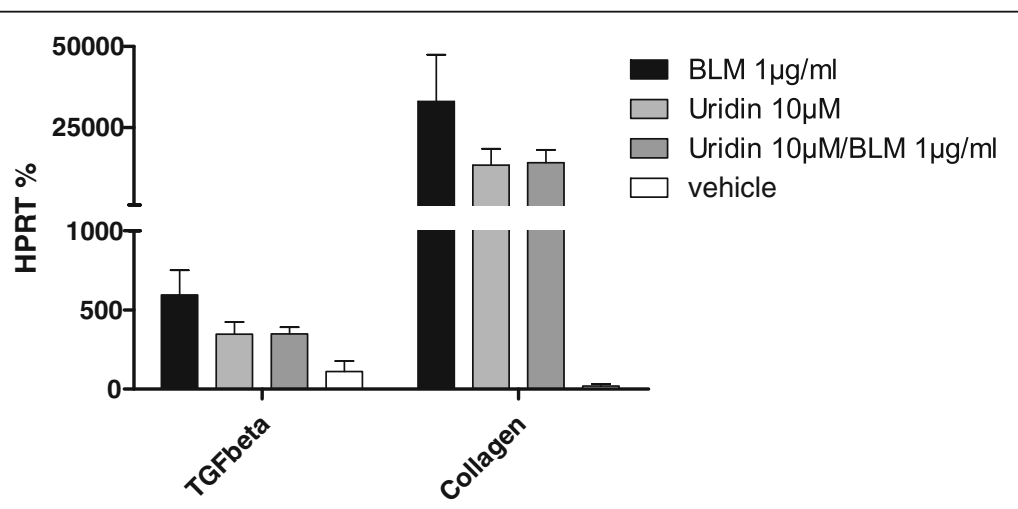

Fig. 5 Uridine inhibits collagen and TGF- $\beta$ production by primary lung fibroblasts. Fibroblasts were stimulated as indicated for 8 h, total RNA was isolated. The mRNA specific for collagen and TGF- $\beta$ was quantified as described. Data are means \pm SEM, $n=3 .{ }^{*} p<0.05 ;{ }^{* *} p<0.01 ;{ }^{* * *} p<0.001$ 

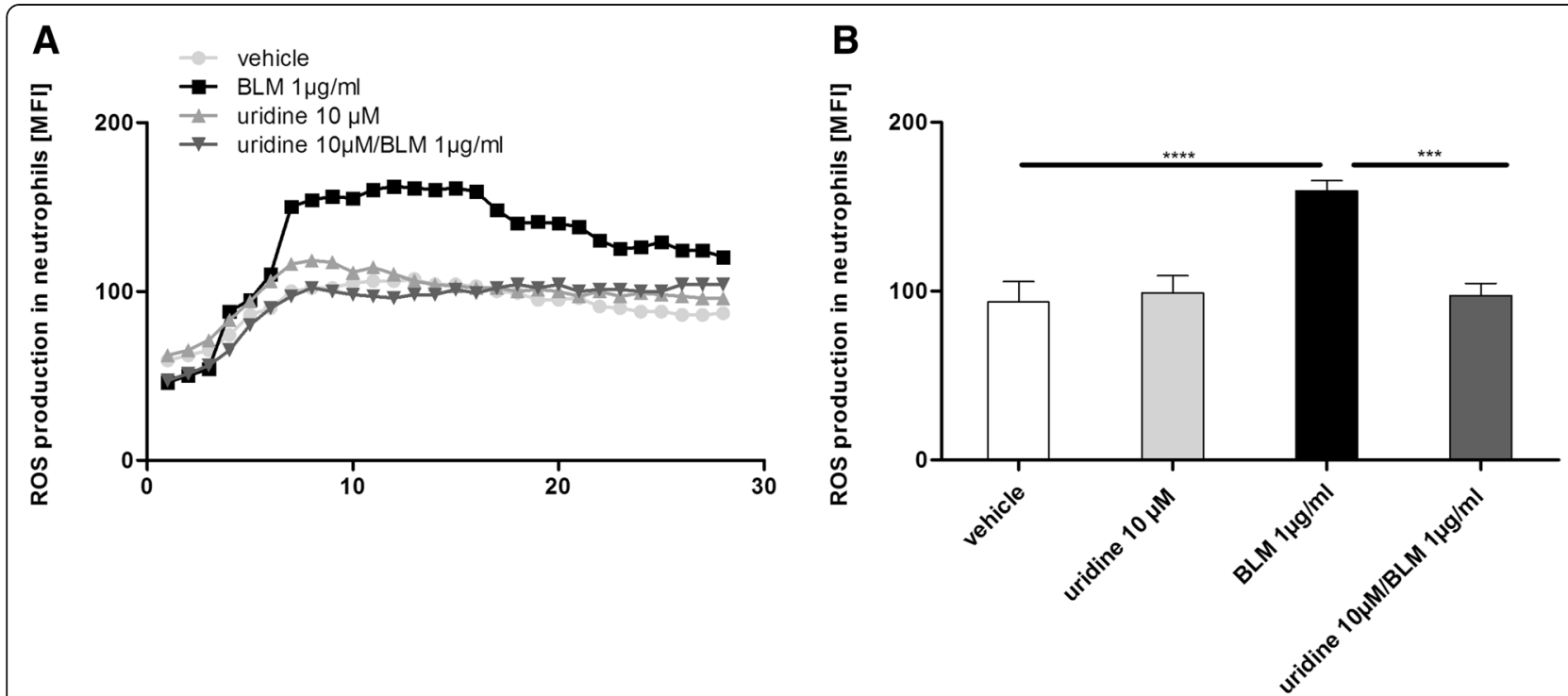

Fig. 6 Uridine inhibits production of reactive oxygen species by human neutrophils. Neutrophils were stimulated as indicated and lucigenin-dependent chemiluminescence was measured. a Time courses upon stimulation with vehicle, bleomycin, and bleomycin + uridine. One representative out of 8 experiments is shown. $\mathbf{b}$ Maximal chemoluminescence after stimulation with vehicle, bleomycin, and bleomycin + uridine. Data are means \pm SEM, $n=7 .{ }^{*} p<0.05 ;{ }^{* *} p<0.01 ;{ }^{* *} p<0.001 ; * * * 0<0,0001$

in adenosine deaminase resulting in increased pulmonary adenosine levels actually leads to a fibrotic lung disease. Additionally, in a previous study we were able to demonstrate that the anti-inflammatory effects of uridine on lung epithelial cells were not dependent on adenosine receptors [8]. Therefore, taken together the anti-inflammatory and anti-fibrotic effects of uridine are most likely independent of adenosine receptors.

The subsequent release of pro-inflammatory and profibrotic cytokines/chemokines after epithelial cell injury is a crucial step in the pathogenesis of fibrotic lung diseases [1]. In this study we were able to demonstrate that when co-administered with bleomycin uridin inhibited the release of the pro-inflammatory cytokines IL- 6 and IL-8 by human alveolar epithelial cells. The latter one is considered as the key chemokine for the recruitment and activation of neutrophils [23]. Interestingly, an association between a high BAL fluid neutrophil count and a poor prognosis in IPF has been found [24]. Apart from inflammation IL-8 has been identified as a pro-angiogenic factor in the context of idiopathic pulmonary fibrosis [25]. IL-6 is a pleiotropic cytokine whose importance in various lung disorders including pulmonary fibrosis has been demonstrated extensively $[15,26]$. Hence, uridine might influence the course of fibrotic lung disease by altered cytokine secretion of epithelial cells. However, in vivo altered cytokine secretion after uridine administration might not be limited exclusively to airway epithelial cells and indirect effects of uridine might also be of importance. In a line with these findings, uridine was also able to decrease TGF- $\beta$ and collagen synthesis by primary lung fibroblasts. The production and deposition of collagen and other extracellular matrix components is a characteristic feature of pulmonary fibrosis. In this context TGF- $\beta$ has been identified as an important growth factor, mediating e.g. epithelial to mesenchymal transition [27].

Though the exact role of oxygen radicals in the pathophysiology of IPF remains controversial increased markers of oxidative stress have been found in IPF patients [28]. Thus decreased production of reactive oxygen species might contribute to reduced pulmonary inflammation and fibrosis in uridine treated animals.

As mentioned earlier in this manuscript treatment with uridine has been shown to abrogate mitochondrial toxicity induced by anti-retroviral therapy in patients infected with HIV [10, 11]. Recently, mitochondrial dysfunction has gained attention as a mechanism contributing to epithelial cell injury and the progression of pulmonary fibrosis $[29,30]$. Hence, uridine supplementation might lead to an improved mitochondrial function thereby limiting pulmonary inflammation and fibrosis.

Nucleosides are rapidly metabolized in the extracellular space. Adenosine, e.g. is characterized by an extremely short half-life when administered intravenously [31]. Hence the pharmacokinetic characteristics of uridine have to be taken in account in this context. However, previous studies were able to demonstrate that the half-life of uridine in plasma is considerably higher compared to adenosine [32, 33]. Accordingly, uridine plasma levels after intraperitoneal administration appear to be high enough to exert anti-inflammatory and anti-fibrotic effects. 
In summary we demonstrated that uridine inhibits inflammation and fibrosis in bleomycin induced lung injury. As a receptor specific for uridine has not been identified yet, the exact mechanisms behind these findings are not completely understood at the moment. Nevertheless, uridine inhibited the production of collagen and proinflamamtory/fibrotic cytokines by alveolar epithelial cells and lung fibroblasts, as well as the release of reactive oxygen species by activated neutrophils. Interestingly, uridine supplementation has been found to be safe and well tolerated in humans $[10,11]$. Therefore, further studies investigating the impact of uridine administration on the course of fibrotic lung diseases in humans would be of great interest.

\section{Conclusions}

Uridine which can be safely administered in humans is a potent inhibitor of bleomycin-induced pulmonary inflammation and fibrosis. Consecutively, though further research is still needed, this might be a new approach for the treatment of fibrotic lung diseases in humans.

\section{Competing interests}

The authors declare that they have no competing interests.

\section{Authors' contributions}

Experiments were designed by $\mathrm{MI}$ and TM. Experiments were performed and analyzed by SC, MG, KA, JB, GZ, AM, MH and TM. The manuscript was written by SC, TM and MI. All authors read and approved the final manuscript.

\section{Acknowledgements}

This work was supported by a Grant from the German Research Foundation (DFG) to TM (Grant ID MU 3194/2-1).

\section{Author details}

${ }^{1}$ Department of Pneumology, University Hospital Freiburg, Killianstrasse 6, 79106 Freiburg, Germany. ${ }^{2}$ Department of Internal Medicine I, University Hospital RWTH Aachen, Aachen, Germany.

Received: 31 March 2015 Accepted: 24 August 2015

Published online: 15 September 2015

References

1. King TE, Pardo A, Selman M. Idiopathic pulmonary fibrosis. Lancet. 2011;378:1949-61.

2. Wolters PJ, Collard HR, Jones KD. Pathogenesis of idiopathic pulmonary fibrosis. Annu Rev Pathol. 2014;9:157-79.

3. Wuyts WA, Agostini C, Antoniou K, Bouros D, Chambers R, Cottin V, et al. The pathogenesis of pulmonary fibrosis: a moving target. Eur Respir J Off J Eur Soc Clin Respir Physiol. 2012;41(5):1207-18.

4. Blackburn MR, Lee CG, Young HWJ, Zhu Z, Chunn JL, Kang MJ, et al. Adenosine mediates IL-13-induced inflammation and remodeling in the lung and interacts in an IL-13 - adenosine amplification pathway. J Clin Invest. 2003;112:332-44

5. Da Rocha Lapa F, de Oliveira APL, Accetturi BG, de Oliveira Martins I, Domingos HV, de Almeida Cabrini D, et al. Anti-inflammatory effects of inosine in allergic lung inflammation in mice: evidence for the participation of adenosine A(2A) and A (3) receptors. Purinergic Signal. 2013;9(3):325-36.

6. Liaudet L, Mabley JG, Pacher P, Virág L, Soriano FG, Marton A, et al. Inosine exerts a broad range of antiinflammatory effects in a murine model of acute lung injury. Ann Surg. 2002;235:568-78.

7. Evaldsson C, Rydén I, Uppugunduri S. Anti-inflammatory effects of exogenous uridine in an animal model of lung inflammation. Int Immunopharmacol. 2007;7:1025-32.
8. Müller T, Grimm M, de Vieira RP, Cicko S, Dürk T, Sorichter S, et al. Local administration of uridine suppresses the cardinal features of asthmatic airway inflammation. Clin Exp Allergy. 2010;40:1552-60.

9. Uppugunduri S, Gautam C. Effects of uridine, isomatitol and 4-thiouridine on in vitro cell adhesion and in vivo effects of 4-thiouridine in a lung inflammation model. Int Immunopharmacol. 2004;4:1241-8.

10. McComsey GA, Walker UA, Budhathoki CB, Su Z, Currier JS, Kosmiski L, et al. Uridine supplementation in the treatment of HIV lipoatrophy: results of ACTG 5229. AIDS. 2010;24:2507-15.

11. Venhoff N, Lebrecht D, Deveaud C, Beauvoit B, Bonnet J, Müller K, et al. Oral uridine supplementation antagonizes the peripheral neuropathy and encephalopathy induced by antiretroviral nucleoside analogues. AIDS. 2010;24:345-52.

12. Cicko S, Lucattelli M, Müller T, Lommatzsch M, De Cunto G, Cardini S, et al. Purinergic receptor inhibition prevents the development of smoke-induced lung injury and emphysema. J Immunol. 2010;185:688-97.

13. Lommatzsch M, Cicko S, Müller T, Lucattelli M, Bratke K, Stoll P, et al. Extracellular adenosine triphosphate and chronic obstructive pulmonary disease. Am J Respir Crit Care Med. 2010;181:928-34.

14. Chua F, Gauldie J, Laurent GJ. Pulmonary fibrosis: searching for model answers. Am J Respir Cell Mol Biol. 2005;33:9-13.

15. Pedroza M, Schneider DJ, Karmouty-Quintana H, Coote J, Shaw S, Corrigan $\mathrm{R}$, et al. Interleukin- 6 contributes to inflammation and remodeling in a model of adenosine mediated lung injury. PLoS One. 2011;6:e22667.

16. Young HWJ, Molina JG, Dimina D, Zhong H, Jacobson M, Chan L-NL, et al. $\mathrm{A} 3$ adenosine receptor signaling contributes to airway inflammation and mucus production in adenosine deaminase-deficient mice. J Immunol. 2004;173:1380-9

17. Evaldsson C, Rydén I, Rosén A, Uppugunduri S. 4-Thiouridine Induces Dose-Dependent Reduction of Oedema, Leucocyte Influx and Tumour Necrosis Factor in Lung Inflammation. Clin Exp Immunol. 2009;155:330-8.

18. Müller T, Robaye B, Vieira RP, Ferrari D, Grimm M, Jakob T, et al. The purinergic receptor $\mathrm{P} 2 \mathrm{Y} 2$ receptor mediates chemotaxis of dendritic cells and eosinophils in allergic lung inflammation. Allergy. 2010;65:1545-53.

19. Vieira RP, Müller T, Grimm M, von Gernler V, Vetter B, Dürk T, et al. Purinergic receptor type 6 contributes to airway inflammation and remodeling in experimental allergic airway inflammation. Am J Respir Crit Care Med. 2011;184:215-23.

20. Yilmaz MS, Coskun C, Suzer O, Yalcin M, Mutlu D, Savci V. Hypotensive effects of intravenously administered uridine and cytidine in conscious rats: involvement of adenosine receptors. Eur J Pharmacol. 2008;584:125-36.

21. Brown RA, Spina D, Page CP. Adenosine receptors and asthma. Br J Pharmacol. 2008;153 Suppl(December 2007):S446-56

22. Polosa R, Blackburn MR. Adenosine receptors as targets for therapeutic intervention in asthma and chronic obstructive pulmonary disease. Trends Pharmacol Sci. 2009;30:528-35.

23. Baggiolini M, Walz A, Kunkel SL. Neutrophil-activating peptide-1/ interleukin 8, a novel cytokine that activates neutrophils. J Clin Invest. 1989;84:1045-9.

24. Kinder BW, Brown KK, Schwarz MI, Ix JH, Kervitsky A, King TE. Baseline BAL neutrophilia predicts early mortality in idiopathic pulmonary fibrosis. Chest. 2008;133:226-32

25. Keane MP, Arenberg DA, Lynch JP, Whyte RI, lannettoni MD, Burdick MD, et al. The CXC chemokines, IL-8 and IP-10, regulate angiogenic activity in idiopathic pulmonary fibrosis. J Immunol. 1997;159:1437-43.

26. Saito F, Tasaka S, Inoue K-I, Miyamoto K, Nakano Y, Ogawa Y, et al. Role of interleukin-6 in bleomycin-induced lung inflammatory changes in mice. Am J Respir Cell Mol Biol. 2008:38:566-71.

27. Sureshbabu A, Tonner E, Allan GJ, Flint DJ. Relative Roles of TGF- $\beta$ and IGFBP-5 in Idiopathic Pulmonary Fibrosis. Pulm Med. 2011;2011:517687.

28. Kliment CR, Oury TD. Oxidative stress, extracellular matrix targets, and idiopathic pulmonary fibrosis. Free Radic Biol Med. 2010;49:707-17.

29. Bueno M, Lai Y-C, Romero Y, Brands J, St Croix CM, Kamga C, et al. PINK1 deficiency impairs mitochondrial homeostasis and promotes lung fibrosis. J Clin Invest. 2015;125:521-38.

30. Patel AS, Song JW, Chu SG, Mizumura K, Osorio JC, Shi Y, et al. Epithelial cell mitochondrial dysfunction and PINK1 are induced by transforming growth factor-beta1 in pulmonary fibrosis. PLoS One. 2015;10:e0121246. 
31. Uematsu T, Kozawa O, Matsuno H, Niwa M, Yoshikoshi H, Oh-uchi M, et al. Pharmacokinetics and tolerability of intravenous infusion of adenosine (SUNY4001) in healthy volunteers. Br J Clin Pharmacol. 2000;50:177-81.

32. Kim E, Kang W. Pharmacokinetics of uridine following ocular, oral and intravenous administration in rabbits. Biomol Ther (Seoul). 2013;21:170-2.

33. Weinberg ME, Roman MC, Jacob P, Wen M, Cheung P, Walker UA, et al. Enhanced uridine bioavailability following administration of a triacetyluridine-rich nutritional supplement. PLoS One. 2011;6:e14709.

Submit your next manuscript to BioMed Central and take full advantage of:

- Convenient online submission

- Thorough peer review

- No space constraints or color figure charges

- Immediate publication on acceptance

- Inclusion in PubMed, CAS, Scopus and Google Scholar

- Research which is freely available for redistribution 